\title{
Ventriculoperitoneal shunts for treating increased intracranial pressure in cryptococcal meningitis with or without ventriculomegaly
}

\author{
Marcelo Corti ${ }^{[1],[2]}$, Maria Priarone ${ }^{[1]}$, Ricardo Negroni ${ }^{[3]}$, Leonardo Gilardi ${ }^{[4]}$, \\ Jimena Castrelo ${ }^{[1]}$, Alicia Irene Arechayala $a^{[3]}$, Fernando Messina ${ }^{[3]}$ \\ and Osvaldo Franze ${ }^{[5]}$
}

[1]. School of Medicine, University of Buenos Aires, Buenos Aires, Argentina. [2]. HIV/AIDS Division, Francisco Javier Muñiz Hospital, Buenos Aires, Argentina. [3]. Mycology Unit, Francisco Javier Muñiz Hospital, Buenos Aires, Argentina. [4]. Sociedad Iberoamericana de Información Cientifica, Buenos Aires, Argentina.

[5]. Neurosurgery Department, Francisco Javier Muñiz Hospital, Buenos Aires, Argentina.

\begin{abstract}
Introduction: Cryptococcosis is an opportunistic mycosis, especially in patients that are human immunodeficiency virus (HIV)-positive, and frequently involves the central nervous system. Methods: We assessed the potential of ventriculoperitoneal shunting (VPS) in preventing mortality due to uncontrollable intracranial hypertension (ICH) in 15 patients with acquired immunodeficiency syndrome (AIDS)-related cryptococcal meningitis. Results: After 2 weeks of antifungal therapy consisting of amphotericin B deoxycholate with or without fluconazole, patients with persistent ICH underwent VPS, despite having persistent Cryptococcus neoformans infection. In 12 patients, the uncontrollable ICH was resolved by VPS. Conclusions: Patients with cryptococcal meningoencephalitis who have ICH must be considered for VPS even with positive cerebrospinal fluid cultures.
\end{abstract}

Keywords: Cryptococcus neoformans. Cryptococcal meningitis. Intracranial hypertension. Ventriculoperitoneal shunts.

Neurocryptococcosis is a fungal illness with high mortality and represents the second most common infection of the central nervous system in patients with acquired immunodeficiency syndrome (AIDS). The incidence of the infection caused by Cryptococcus in patients with AIDS is related to other factors beyond a low level of the cluster of differentiation 4 (CD4) T-cell counts and the fungal exposure.

Cryptococcus neoformans is the etiologic agent of cryptococcosis, a major opportunistic infection in patients with impaired cellular immune response, especially those with AIDS and diabetes mellitus. C. neoformans capsule is considered as the major virulence factor of this intracellular pathogen. The polysaccharide capsule antigen contributes to the virulence by being antiphagocytic among other features that affect the host immune response ${ }^{1,2}$.

Cryptococcal meningitis treatment has been primarily focused on optimizing antifungal therapy based on amphotericin B plus flucytosine or fluconazole. However, elevated intracranial pressure is an important risk factor for high mortality in this opportunistic infection in immunosuppressed patients,

Address to: Dr. Marcelo Corti. Infectious Diseases F.J. Muñiz Hospital. Puán 381/2 floor, C1406 CQG Buenos Aires, Argentina.

Phone: 5411 4432-3762

e-mail: marcelocorti@fibertel.com.ar

Received 5 September 2013

Accepted 14 May 2014 especially those with human immunodeficiency virus/acquired immunodeficiency syndrome (HIV/AIDS) disease ${ }^{1}$. In this study, we evaluate, retrospectively, the benefits of ventriculoperitoneal (VP) shunting in order to improve the survival rate of patients with uncontrollable intracranial hypertension (ICH) associated with cryptococcal meningitis.

We retrospectively analyzed the epidemiological (age, gender, risk factors for HIV infection), clinical, microbiological, and immunological characteristics, and clinical outcomes of 15 patients who developed cryptococcal meningitis and presented symptoms consistent with ICH including headache, altered mental status, meningism, and neurosensory changes. Clinical and neurological examinations were performed on all patients. To evaluate the inflammatory response to the fungal infection, all samples of cerebrospinal fluid (CSF) were analyzed for white blood cell counts, glucose, and protein levels. Direct examination with India ink stain and cultures on Sabouraud dextrose agar and brain-hart infusion agar were also performed. Before lumbar puncture, a head computed tomography (CT) scan was done in all cases. CD4 T cell counts were taken from all patients with HIV. Elevated intracranial pressure was defined as $\geq 25 \mathrm{~cm} \mathrm{H}_{2} \mathrm{O}$ of CSF during lumbar puncture. Patients with persistent elevated spinal pressure following frequent lumbar puncture drainage and having undergone 2 weeks of cumulative amphotericin B deoxycholate therapy with or without fluconazole underwent a VP shunt $\left(\right.$ Medtronic $^{\circledR}$ ), despite positive CSF cultures for C. neoformans. Differentiation of the encapsulated yeast, $C$. neoformans, was performed using the creatine-dextrose-bromothymol-blue-thymine (CDBT) 
agar medium. The isolates were identified as $C$. neoformans or $C$. gattii by urease production in Christensen-agar medium, evidence of phenol-oxidase in sunflower seed-agar, and growth capacity at $37^{\circ} \mathrm{C}$. Both species were differentiated using glycinecanavanine-bromothymol blue agar medium. Clinical outcomes included mortality rate and sequelae, and complete responses were reported. All available data were obtained from medical records and analyzed with descriptive statistical tests, including the description of measures of central tendency, and variability or dispersion. Microsoft Excel ${ }^{\circledR} 2007$ was used for data analysis.

Fifteen patients ( 10 male and 5 female; median age, 33 years; range, 18-53 years) with cryptococcal meningitis-associated ICH were included. All of them presented disseminated cryptococcosis with meningeal involvement. They suffered altered mental status, meningism, headache, and visual/hearing changes. Risk factors for cryptococcal meningitis included HIV/AIDS disease in 14 cases and diabetes mellitus type-2 in one patient. Risk factors for HIV infection were available in 12 patients and included unprotected homosexual contact [3 (21\%) patients] and unprotected heterosexual contact [9 (64\%) patients]. Demographic findings are included in Table 1. All patients were seronegative for hepatitis $\mathrm{C}$ virus ( $\mathrm{HCV}$ ) infection. Headache and fever were present in all patients. In the $14 \mathrm{HIV}$ seropositive patients, the median of CD4 $\mathrm{T}$ cell count at the time of neurocryptococcosis diagnosis was 50 cells $/ \mu \mathrm{L}$ (range, 6-511 cells $/ \mu \mathrm{L}$ ). At baseline, all CSF samples were macroscopically clear. All patients had an opening pressure higher than $25 \mathrm{~cm}$ $\mathrm{H}_{2} \mathrm{O}$, with a median of $42 \mathrm{~cm} \mathrm{H}_{2} \mathrm{O}$ (range, 26-50 $\mathrm{cm} \mathrm{H}_{2} \mathrm{O}$ ). The median glucorrachia level was $40 \mathrm{mg} / \mathrm{dL}$ (range, 11-70mg/ $\mathrm{dL}$ ). Mean CSF protein levels was $1.0 \mathrm{~g} / \mathrm{dL}$ with a median of $0.67 \mathrm{mg} / \mathrm{dL}$ (range, $0.28-2.74 \mathrm{mg} / \mathrm{dL}$ ). Median leukocyte count in CSF was 55 cells $/ \mu \mathrm{L}$ (range, 1-690cells $/ \mu \mathrm{L}$ ), most of which were lymphocytes and monocytes. Direct examination of CSF with India ink smear was positive in $75 \%$ of patients; cultures were positive in $100 \%$ of them. C. neoformans var. grubii was identified in all cases in which the CDBT test was performed.
Before surgical procedure, the patients began antifungal therapy based on amphotericin B deoxycholate alone or in combination with fluconazole and continued with the frequent high-volume lumbar puncture if they were neurologically symptomatic. At preoperative time, the median opening pressure was $42 \mathrm{~cm} \mathrm{H}_{2} \mathrm{O}$ (range, $15-65 \mathrm{~cm} \mathrm{H}_{2} 0$ ). Preoperative cultures of CSF were positive in $50 \%$ of patients. Polysaccharide cryptococcal capsular antigen in CSF was positive in all cases, with a median titer of $1 / 1,000$ (range, $1 / 10$ to $1 / 5,000$ ). Serum polysaccharide cryptococcal capsular antigen was also positive in all of the patients, with a median titer of $1 / 550$ (range, $1 / 10$ to $1 / 10,000$ ).

All patients underwent implantation of CSF shunts; 14 patients received VP shunts and one other was treated with a lumbar-peritoneal derivation. Twelve patients had good clinical outcomes; three presented complications related to the neurosurgical procedure. This included sepsis with meningitis and multiorgan failure in one of the patients who died, postoperative bacterial meningitis with a good response to antibiotic therapy, and a large abdominal multiseptated pseudocyst which required the VP shunt removal. Patients who had complications related to the surgical procedure were excluded from the postoperative analysis. One month after surgery, considered as the postoperative period, CSF median aperture pressure was sharply reduced in the 12 patients, with a median of $14.5 \mathrm{~cm} \mathrm{H}_{2} \mathrm{O}$ (range: 10 to $20 \mathrm{~cm} \mathrm{H}_{2} \mathrm{O}$ ) and only two (16\%) patients persisted with positive CSF cultures. All biochemical and microbiological parameters of the CSF were also modified in these patients, with a dramatical reduction in the indirect signs of cryptococcal infection (antigenorrachia and antigenemia titers) and inflammatory response. Glucorrachia was not modified after VPS and showed a median of $41 \mathrm{mg} /$ $\mathrm{dL}$ (range, $38-85 \mathrm{mg} / \mathrm{dL}$ ). Median cell counts decreased to 3.5 cells $/ \mu \mathrm{L}$ (range, $1-40$ cells $/ \mu \mathrm{L}$ ), while proteinorrachia slightly increased as expected owing to the neurosurgery itself, with a median of $0.89 \mathrm{~g} / \mathrm{dL}$ (range, $0.33-2.6 \mathrm{~g} / \mathrm{dL}$ ). Polysaccharide cryptococcal capsular antigen in CSF was positive in $90 \%$ of cases, with a median titer of $1 / 550$ (range, $1 / 10$ to $1 / 1,000$ ).

TABLE 1 - Demographic data of 15 patients with ICH associated with cryptococcal meningitis.

\begin{tabular}{|c|c|c|c|}
\hline & Whole population & Male & Female \\
\hline Age (median, years) & 33.5 & 34.5 & 31.5 \\
\hline \multirow[t]{2}{*}{ Risk factor for cryptococcosis } & HIV/AIDS (14; 93\%) & - & - \\
\hline & Diabetes $(1 ; 7 \%)$ & - & - \\
\hline \multirow{2}{*}{ Risk factor for HIV (14 patients) } & Unp hom cont $(\mathrm{n}=3 ; 21 \%)^{* *}$ & - & - \\
\hline & No data $(\mathrm{n}=2 ; 15 \%)$ & - & - \\
\hline \multirow[t]{2}{*}{$\mathrm{CD} 4+($ median, cells $/ \mu \mathrm{L})$} & 50 & - & - \\
\hline & (range: 6 to 511 ) & - & - \\
\hline
\end{tabular}

ICH: intracranial hypertension; HIV/AIDS: human immunodeficiency virus/acquired immunodeficiency syndrome; CD4: cluster of differentiation 4. *Unprotected heterosexual contact. **Unprotected homosexual contact. 
Serum polysaccharide cryptococcal capsular antigen was also positive in $90 \%$ of patients, with a median titer of $1 / 100$ (range, 0 to $1 / 10,000$ ). Even though the small sample size precludes an optimal statistical analysis of these findings, median CSF aperture pressure was significantly reduced $(\mathrm{p}<0.01)$. Microbiological data for baseline, preoperative, and one month postoperative are summarized in Table 2. Neurosensory sequelae were frequent in patients and included different grades of visual loss (including blindness) in six and hearing loss in two. Mean complete dose of amphotericin B after surgery was 3,000mg; median complete dose was 1,880mg (range, $250-5,650 \mathrm{mg}$ ). Higher doses were not associated with better outcome of neurosensory sequelae or postsurgical complications. After a minimum of one year of follow up, 12 patients were still alive and negative for C. neoformans upon microbiological examination of CSF.

Cryptococcal meningitis is one of the most common fungal infections in AIDS patients. The treatment of this opportunistic infection remains sub-optimal in many settings. Among patients with AIDS, the rate of infection ranges from $23 \%$ to $48.6 \%$ and neurocryptococcosis is the most frequent clinical manifestation of the disease in $70 \%$ to $90 \%$ of all the cases ${ }^{2,3}$. Patients with AIDS having cryptococcal meningitis differ from non-HIVinfected subjects with the same infection in that they present very little inflammatory response in CSF, large fungal burden, and few mass lesions.

The most severe complication of this opportunistic disease is the uncontrollable ICH. Uncontrollable ICH due to cryptococcal meningitis in patients with AIDS plays an important role in morbidity and mortality ${ }^{14}$. In a recent study, Souza et al. ${ }^{15}$ reported a high crytococcal meningoencephalitis-related mortality (48.4\%) in Brazil. The pathophysiology of ICH in these patients is poorly understood. Fries and colleagues, suggest that strain-specific characteristics can influence in the development of ICH in an experimental model of rats with cryptococcal meningoencephalitis. The authors found that phenotypic variants of $C$. neoformans (smooth and mucoid) differed in their abilities to promote ICH. In their experimental study, rats infected with the mucoid variant develop ICH whereas rats infected with the smooth phenotype did not. These results correlated with a short survival and a higher CSF fungal burden in mucoid variant-infected rats. In addition, higher levels of polysaccharide accumulated in the CSF and brain of mucoid variant-infected rats were detected. These findings were also associated with a more pronounced but ineffective inflammatory response in the meninges of mucoid-variant of C. neoformans.

The mechanisms underlying ICH in patients with cryptococcal meningitis include increased vascular permeability and cerebral edema associated with cytokine-induced inflammation and the clogging of the arachnoid villi with cryptococci and the consecutive impairment of their resorptive function ${ }^{4}$. Beside these, $C$. neoformans also has several virulence factors such as urease, mannitol, and inositol, which have been implicated in increasing brain edema and CSF osmolarity ${ }^{4}$.

In a large and prospective clinical trial, Graybill and colleagues ${ }^{5}$ demonstrated that higher CSF opening pressure at baseline was correlated with higher cryptococcal capsular polysaccharide antigen titers, a higher frequency of positive India ink smear results, and a higher frequency of headache and neurological compromise. In addition, patients with ICH had a shorter life span than those who had baseline-opening pressures $<250 \mathrm{~mm} \mathrm{H}_{2} \mathrm{O}$.

Different studies suggest that the opening pressure should be measured at the first lumbar puncture in all patients with suspected cryptococcal meningitis. In those with elevated intracranial pressure, additional lumbar punctures, including daily lumbar punctures, should be performed until the pressure is adequately controlled ${ }^{6}$. Pharmacological agents such as mannitol and corticosteroids are generally avoided, and neurological interventions, including VP or ventriculoatrial shunts placement, are generally delayed or avoided ${ }^{7}$.

Among patients with uncontrollable ICH, the best time to implant a permanent shunt (VP or ventriculoatrial) is controversial, but generally, the device must be placed after the initiation of an optimal antifungal therapy?

TABLE 2 - Cerebrospinal fluid data in 15 patients with ICH associated with cryptococcal meningitis.

\begin{tabular}{|c|c|c|c|}
\hline & Baseline & Preoperative & Postoperative \\
\hline Glucorrachia (median, mg/dL) & 40 & - & 41 \\
\hline Proteinorrachia (median, g/dL) & 0.67 & - & 0.895 \\
\hline CSF cell count (median, cells $/ \mu \mathrm{L}$ ) & 55 & - & 3.5 \\
\hline CSF culture (\%) & 100 & 50 & 10 \\
\hline Antigenorrachia (median, titer) & - & $\begin{array}{c}1 / 1,000 \\
\text { Positivity: } 100 \%\end{array}$ & $\begin{array}{c}\text { 1/55 } \\
\text { Positivity: } 90 \%\end{array}$ \\
\hline Antigenemia (median, titer) & - & $\begin{array}{c}1 / 550 \\
\text { Positivity: } 100 \%\end{array}$ & $\begin{array}{c}\text { 1/100 } \\
\text { Positivity: } 90 \%\end{array}$ \\
\hline
\end{tabular}

CSF: cerebrospinal fluid; ICH: intracranial hypertension. 
Mechanical drainage of CSF is a part of managing ICH in cryptococcal meningitis associated with AIDS. Some authors describe important sequelae, probably related to the ischemia resulting from $\mathrm{ICH}^{8}$. Serial lumbar punctures are a common practice among infectologists and clinicians for the management of these patients. As a secondary choice, some clinicians prefer to place a lumbar drainage, which has a high risk of infection or malfunction ${ }^{8}$. The need for permanent VP shunting after 4 weeks of appropriate antifungal therapy is often necessary in patients who require serial lumbar punctures ${ }^{9}$.

CSF culture is one of the methods to predict the clinical outcome in these patients. Robinson and colleagues ${ }^{10}$ suggest that a positive CSF culture for C. neoformans after 2 weeks of antifungal therapy is associated with a poor clinical outcome in comparison with a negative result. Elevated intracranial pressure is another significant and predictive factor for morbidity and mortality in patients with HIV-associated cryptococcal meningitis ${ }^{9}$. Patients who present high intracranial pressure that persist despite antifungal therapy based on amphotericin B plus fluconazole or flucytosine, have a high risk of serious sequelae and mortality rates. These patients include those who do not respond adequately to antifungal therapy, remain with high CSF pressure levels, and have persistent neurological symptoms despite frequent lumbar punctures ${ }^{10}$. They should be considered for CSF shunting at an early stage of the disease.

ICH is associated with serious complications in patients with cryptococcal meningitis and can be adequately treated with the placement of a VP shunt, even in those cases where the neuroimaging studies do not reveal hydrocephalus and those of persistent CSF cryptococcal infection ${ }^{11,12}$.

Although the medical management of ICH in these patients is discussed, the placement of a VP shunt should be the primary therapeutic alternative ${ }^{13}$. However, post-surgical meningitis and ventriculitis from shunt infection are well-documented complications in these patients. Both complications frequently occur in the early postoperative stage and are secondary to wound infection or intraoperative contamination. Acute visceral perforation leading to peritonitis by the abdominal portion of VP shunt is a rare but severe complication of this procedure. Another possible complication is the formation of loculated intraperitoneal pseudocysts with infected or sterile CSF that are associated with abdominal, gastrointestinal, or genitourinary symptoms. It is generally assumed that device implantation on a non-sterile medium may be associated with clinical progression and device infection. However, clinical conditions and potential or established complications, like ICH, may still be considered for device implantation. In a risk versus benefits assessment, VP shunts may be useful in selected patients independent of the persistence of cryptococcal infection, in order to prevent sequelae or to optimize the management of intracranial pressure. In this regard, in our study that includes immunocompromised patients, we identified only three complications associated with the surgical procedure.

There are obvious limitations to this study, including its retrospective nature and the obvious absence of a control group. The small size of the cohort and the participation of a single center of infectious diseases are other limitations.
In conclusion, patients who do not benefit from antifungal therapy alone, remain with high CSF pressure levels, and continue with neurological symptoms despite frequent lumbar punctures, must be considered for VP placement. In particular, VP placement must be considered in those who present serious visual loss or ocular palsies, even in cases of persistent CSF cryptococcal infection. Early diagnosis and shunt placement are essential to improve the poor survival rate and the neurological functions in these critically ill patients.

\section{CONFLICT OF INTEREST}

The authors declare that there is no conflict of interest.

\section{REFERENCES}

1. Shoham S, Cover C, Donegan N, Fulnecky E, Kumar P. Cryptococcus neoformans meningitis at 2 hospitals in Washington, D.C.: adherence of health care providers to published practice guidelines for the management of cryptococcal disease. Clin Infect Dis 2005; 40:477-479.

2. Corti M, Villafañe MF, Negroni R, Arechavala A, Maiolo E. Magnetic Resonance Imaging findings in patients with AIDS and central nervous system cryptococcosis. Rev Iberoam Micol 2008; 25:211-214.

3. Satishchandra P, Mathew T, Gadre G, Nagarathna S, Chandramukhi A, Mahadevan A. Cryptococcal meningitis: Clinical, diagnostic and therapeutic overviews. Neurology India 2007; 55:226-231.

4. Fries BC, Lee SC, Kennan R, Zhao W, Casadevall A, Goldman DL. Phenotypic switching of Cryptococcus neoformans can produce variants that elicit increased intracranial pressure in a rat model of cryptococcal meningoencephalitis. Infect Immun 2005; 73:1779-1787.

5. Graybill JR, Sobel J, Saag M, van der Horst C, Powderly WG, Cloud G, et al. Diagnosis and management of increased intracranial pressure in patients with AIDS and cryptococcal meningitis. Clin Infect Dis 2000; 30:47-54.

6. Day JN, Chau TT, Wolbers M, Mai PP, Dung NT, Mai NH, et al. Combination antifungal therapy for cryptococcal meningitis. N Engl J Med 2013; 368:1291-1302.

7. Pappas PG. Management cryptococcal meningitis is about handling the pressure. Clin Infect Dis 2005; 40:480-482.

8. Park MK, Hospenthal DR, Bennett JE. Treatment of hydrocephalus secondary to cryptococcal meningitis by use of shunting. Clin Infect Dis $1999 ; 28: 629-633$.

9. Corti M, Solari R, Cangelosi D, Dominguez C, Yampolsky C, Negroni R, et al. Sudden blindness due to bilateral optic neuropathy associated with cryptococcal meningitis in an AIDS patient. Rev Iberoam Micol 2010; 27:207-209.

10. Robinson PA, Bauer M, Leal MAE, Evans SG, Holtom PD, Diamond DM, et al. Early mycological treatment failure in AIDS-associated cryptococcal meningitis. Clin Infect Dis 1999; 28:82-92.

11. Negroni R. Cryptococcosis. Clin Dermatol 2012; 30:599-609.

12. Woodworth GF, Mc Girt MJ, Williams MA, Rigamonti D. The use of ventriculoperitoneal shunts for uncontrollable intracranial hypertension without ventriculomegally secondary to HIV-associated cryptococcal meningitis. Surg Neurol 2005; 63:529-531.

13. Perfect JR, Dismukes WE, Dromer F, Graybill JR, Hamill RJ, Harrison TS, et al. Clinical practice guidelines for the management of cryptococcal disease: 2010 update by the Infectious Disease Society of America. Clin Infect Dis 2010; 50:291-322.

14. Bach MC, Tally PW, Godofky EW. Use of cerebrospinal fluid shunts in patients having acquired immunodeficiency syndrome with cryptococcal meningitis and uncontrollable intracranial hypertension. Neurosurgery 1997; 41:1280-1282.

15. Souza LKH, Rodrigues Costa C, Lisboa Fernandes O, Yano Abrão F, Silva TC, Martins Treméa C, et al. Clinical and microbiological features of cryptococcal meningitis. Rev Soc Bras Med Trop 2013; 46:343-347. 